\title{
Multilinguales
}

15 | 2021

Recherches algériennes en sociolinguistique et en littérature : discours scientifique/discours militant, quelles frontières?

\section{Esquisse d'une sociolinguistique de la littérature dans le contexte algérien : enjeux théoriques et positionnements épistémologiques}

Outlining sociolinguistics of literature in the Algerian context: theorical issues and epistemological

\section{OpenEdition}

Journals

Édition électronique

URL : https://journals.openedition.org/multilinguales/5978

DOI : $10.4000 /$ multilinguales.5978

ISSN : 2335-1853

Éditeur

Université Abderrahmane Mira - Bejaia

Référence électronique

Mokhtar Boughanem et Hassiba Benaldi,

Esquisse d'une sociolinguistique de la littérature dans le contexte algérien : enjeux théoriques et 《 positionnements épistémologiques », Multilinguales [En ligne], 15 | 2021, mis en ligne le 15 juin 2021, consulté le 14 février 2022. URL : http:// journals.openedition.org/multilinguales/5978; DOI : https://doi.org/10.4000/multilinguales.5978

Ce document a été généré automatiquement le 14 février 2022.

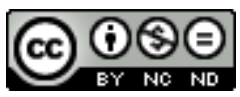

Multilinguales est mise à disposition selon les termes de la Licence Creative Commons Attribution Pas d'Utilisation Commerciale - Pas de Modification 4.0 International 


\section{Esquisse d'une sociolinguistique de la littérature dans le contexte algérien : enjeux théoriques et positionnements épistémologiques}

Outlining sociolinguistics of literature in the Algerian context: theorical issues and epistemological

positions Mokhtar Boughanem et Hassiba Benaldi

1 La présente réflexion part de l'idée selon laquelle la fragmentation institutionnelle des champs disciplinaires rend problématique l'appréhension des objets complexes (Morin, 1990) qui, à l'instar de la langue et de la littérature, requièrent des approches plurielles. En Algérie, les études linguistiques et les études littéraires ont tendance à évoluer de manière autonome les unes des autres. Pourtant, leurs objets respectifs sont appelés à interagir en permanence.

2 La question qui se pose dès lors est de savoir s'il est possible, en l'état actuel des connaissances, de faire dialoguer leurs objets d'étude sans se heurter à de sérieux obstacles épistémologiques. Ainsi que le note Lise Gauvin, « la question des interactions entre langue et littérature, si elle parait au départ affectée d'un caractère d'évidence, se complique dès qu'on y regarde de plus près » (2004:7). D'une part, la langue génère de la littérature sur la base d'une construction discursive rendue possible grâce au processus d'écriture. D'autre part, la littérature procède, au cours de son élaboration, de la déconstruction puis de la reconstruction de la langue. D'où notre objectif de rassembler dans ce travail les présupposés épistémologiques ${ }^{1}$ et théoriques à la lumière desquels l'opposition paradigmatique entre langue et littérature cesse de tourner le dos à la recherche interdisciplinaire. L'intérêt du décloisonnement scientifique ainsi prôné 
réside dans le fait qu'il offre la possibilité d'explorer les zones d'ombre créées sous l'effet du retranchement disciplinaire.

3 Le point de vue que nous adoptons ici est celui d'une sociolinguistique de la littérature qui se veut en construction. Si dans cette perspective la langue peut immédiatement être définie comme un objet social dont les contours sont forgés par les pratiques ainsi que par les représentations des locuteurs, qu'en est-il de la littérature? Quelle réalité socio-langagière recouvre-t-elle? En quoi peut-elle constituer un terrain de recherche pour la sociolinguistique?

Ces questions sont bien sûr à mettre en relation avec le contexte académique algérien. Pour y répondre, nous comptons établir un état des lieux concernant les recherches qui interrogent conjointement les notions de langue et de littérature. A partir de cet état des lieux, il sera question de relever les positionnements épistémologiques adoptés par différents chercheurs algériens.

\section{Une prédisposition à travailler sur des terrains dispersés}

4 La sociolinguistique se donne pour tâche d'explorer les langues dans et à travers leurs contextes d'emploi. Dans cette optique, l'accent est davantage mis sur l'aspect dynamique des faits langagiers à l'œuvre que sur leur aspect statique. Ainsi, conformément à sa vocation, la sociolinguistique est susceptible d'intervenir partout où la langue est employée, partout où des locuteurs se mettent à produire du discours.

C'est ainsi qu'à partir de ce fil conducteur, nous avons pu assister progressivement à la multiplication des terrains de la sociolinguistique. Il peut s'agir de terrains physiques dont la délimitation effective est possible à vue d'œil ou de terrains symboliques au sein desquels se déploie une phénoménologie socio-langagière mouvante. En voici quelques exemples :

- la ville, à travers ses versants linguistique et langagier, constitue l'objet d'étude de la sociolinguistique urbaine (Calvet, 1994);

- l'école est, en ce qu'elle est le siège institutionnel de l'enseignement-apprentissage des langues, le champ d'investigation de la sociolinguistique scolaire (Dabène, 1994);

- le monde du travail, avec ses problématiques de socialisation, d'insertion et de domination, figure au cœur des préoccupations de la sociolinguistique des situations de travail (Boutet, 2008);

- la variation linguistique, en tant que phénomène majeur indissociable de l'activité langagière, est prise en charge par la sociolinguistique variationniste (Gadet, 2007);

- la communication interpersonnelle sous toutes ses formes (interlocution, conversation, échanges, etc.) mobilise autour d'elle la sociolinguistique interactionnelle (Gumperz, 1989);

- les phénomènes liés au métissage linguistique dans les situations bi-plurilingues sont étudiés par la sociolinguistique des contacts de langues (Simonin et Wharton, 2013).

Bien évidemment, cette énumération n’a pas la prétention d'être ni linéaire ni exhaustive. Elle ne fait que mettre en évidence le caractère entropique de la sociolinguistique et sa prédisposition épistémologique à investir, pour peu que la question de la langue soit posée dans un cadre impliquant la dimension sociale, des terrains peu conventionnels.

Partant de cette configuration, nous tenterons le long de cet article d'identifier les ressorts favorables à l'attraction heuristique entre sociolinguistique et littérature. 
Etant à l'opposé de toute vision disjonctive cloisonnée dans la stricte disciplinarité, la sociolinguistique adopte un mode de construction des connaissances de type " hologrammique " (Blanchet, $2015: 66$ ), lequel consiste à considérer les phénomènes langagiers comme étant irréductibles à la somme des parties qui les constituent. Conformément à ce postulat, les interactions qui peuvent être observées entre les parties en question sont à l'origine d'une dynamique d'ensemble qui requiert une démarche constructiviste. Cela suppose pour ce qui nous concerne ici que, une fois mis en contact, les complexes langue et littérature laissent entrevoir des phénomènes émergents qui ne demandent qu'à être explorés en dehors de l'opposition classique dans laquelle ils ont longtemps été confinés. En d'autres termes, la relation entre langue et littérature est à rechercher non pas dans ce qu'est l'une ou l'autre, de manière séparée, mais dans ce qu'implique l'une pour l'autre.

\section{Exploitation des données bibliométriques via la plateforme ASJP}

6 Les données de la plateforme $\mathrm{ASJP}^{2}$ nous ont servi d'indicateurs bibliométriques afin de situer la production scientifique ayant comme objet de réflexion le couple languelittérature. Etant donné que cette plateforme est continuellement alimentée par de nouveaux articles, nous nous sommes contentés de vérifier les données disponibles jusqu'au 31 décembre 2020.

Etant dotée d'un moteur de recherche intégré, la plateforme ASJP nous a permis d'accéder, en un clic, à tous les contenus dans lesquels langue et littérature figurent comme mots-clés ${ }^{3}$. Lors de cette opération de sélection automatique des contenus, nous avons recensé 1065 publications. Après avoir survolé tous les contenus identifiés, nous nous sommes résolus à la nécessité d'effectuer un autre tri manuel, forcément plus rigoureux, de sorte à ne garder que les propositions pertinentes et exploitables. Nous avons ainsi éliminé tous les articles dans lesquels la relation entre langue et littérature n'est pas explicitement abordée ainsi que tous ceux qui ne sont pas rédigés par des chercheurs algériens. A l'issue de cette étape, nous nous sommes retrouvés en présence de 103 articles. Une grille de lecture nous a ensuite permis de repérer, en priorité, la problématique ainsi que l'ancrage théorique de chacun de ces articles.

7 La représentation graphique ci-dessous montre, en termes de pourcentages, la répartition disciplinaire des articles retenus. Il en ressort que, rien que dans le contexte algérien, quatre champs de recherche ${ }^{4}$ 'interrogent sur les spécificités de la relation entre langue et littérature. Ceci témoigne non seulement de l'intérêt scientifique que suscite cette relation, mais aussi des interrogations qu'elle soulève chez de nombreux chercheurs. 
Graphique 1 : Répartition par discipline des recherches traitant de la relation entre langue et littérature

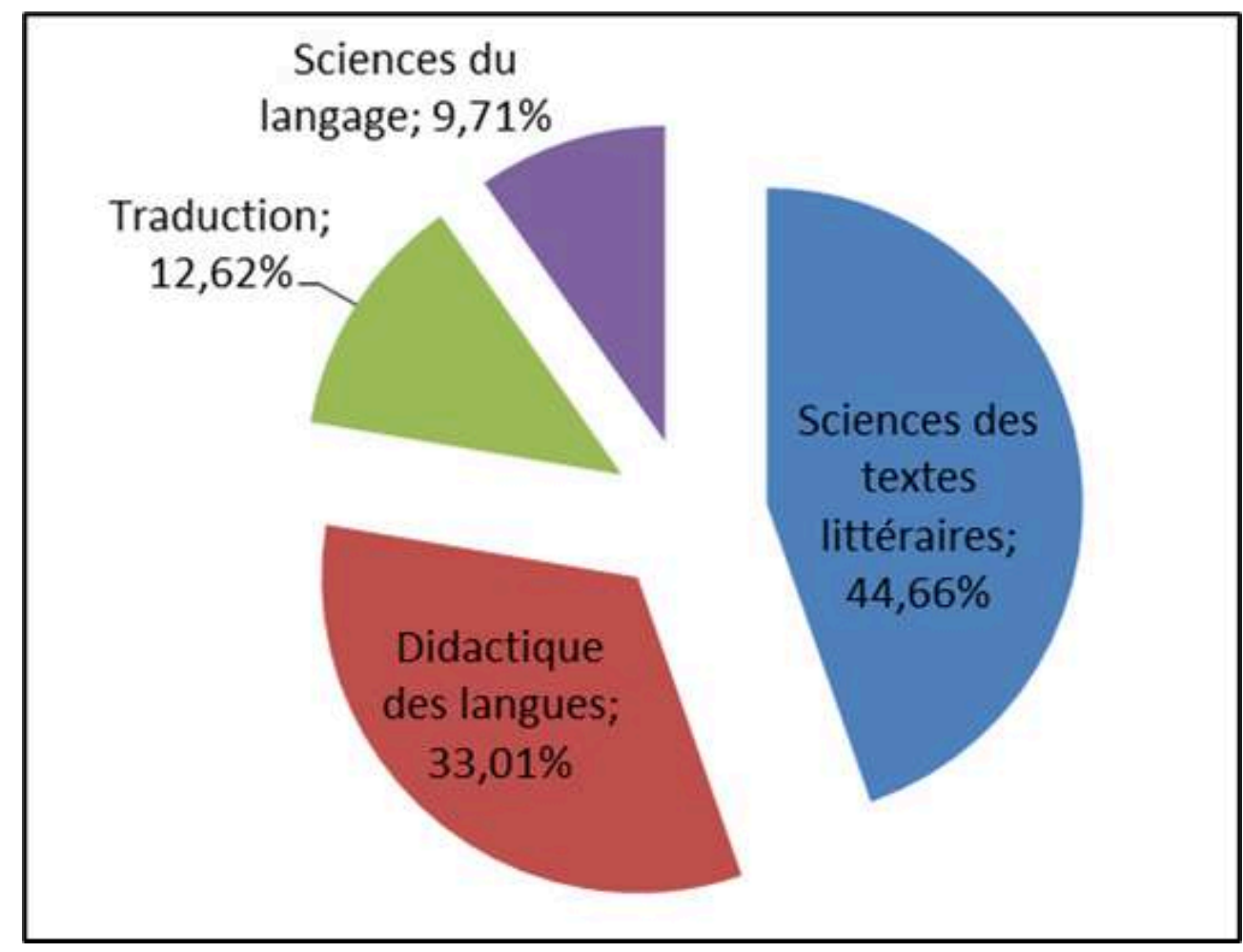

Les chiffres ainsi obtenus ${ }^{5}$ révèlent que, malgré leur grande diversité, les études littéraires n'ont pas la prétention de monopoliser la réflexion sur cette question. Les problématiques développées dans ce sillage portent, entre autres, sur les spécificités linguistiques de l'écriture littéraire, sur les retombées stylistiques liées à l'usage de la langue dans le cadre de la fiction ainsi que sur les modalités d'expression véhiculées par le texte littéraire. Toutes ces orientations sont importantes à prendre en considération dès lors qu'elles sont révélatrices d'un type d'analyse critique à caractère linguistico-interprétatif. Nous y reviendrons plus loin.

La didactique des langues est un autre champ de recherche au sein duquel le texte littéraire est étudié à la lumière de son potentiel didactique en classe. Dans cette optique, le littéraire est, au regard des ressources lexicales, grammaticales et sémantiques qu'il recèle, considéré comme le point de départ pour l'enseignementapprentissage du linguistique. Même s'il n'est représenté que par un taux de $12.62 \%$ dans cette étude statistique, le champ de la traduction n'est pas du tout étranger au débat scientifique engagé autour de la relation entre langue et littérature. La prise en charge de l'opacité culturelle qui sous-tend les écrits littéraires et la gestion des effets de sens lors du passage de la langue source à la langue cible relèvent de ses principales préoccupations.

9 Aux sciences du langage est associé le taux le plus faible (9.71\%) des publications portant sur la relation entre langue et littérature. De notre point de vue, ceci n'est pas le signe d'un désintérêt pour le littéraire, mais d'une méfiance cultivée par une organisation en vase clos des domaines de recherche au sein de l'université algérienne. Ainsi l'étanchéité des frontières disciplinaires favorise-t-elle l'insécurité épistémologique et, par voie de conséquence, le repli sur soi. Les données consultées sur la plateforme ASJP révèlent, néanmoins, qu'au sein des sciences du langage, la 
sociolinguistique et l'analyse du discours sont à l'avant-garde de l'exploration du littéraire.

Dans les paragraphes qui suivent, nous tâcherons d'exposer plus particulièrement les leviers épistémologiques dont se sert la sociolinguistique afin de se frayer un chemin dans le littéraire. Les chiffres maigres enregistrés dans ce cadre témoignent d'une tendance de recherche qui n'en est qu'à ses débuts. D'où notre désir, à travers cette contribution, de documenter quelques aspects saillants inhérents à la genèse d'une réflexion ouverte au dialogue interdisciplinaire. Cependant, avant d'aller plus loin, il est utile de souligner en quoi les études sociolinguistiques se démarquent des études littéraires, notamment à travers les apports et les limites de la glottocritique.

\section{La glottocritique : apports et limites}

Dans les études littéraires, la glottocritique apparait comme l'aboutissement de la réflexion sur la relation entre langue et littérature. Selon Jean Bernabé, cette approche " définit son projet et sa pratique par référence exclusive à la nature ontologiquement langagière des textes» (1982: 85). De plus, celle-ci «intègr[e] dans son propre mouvement l'analyse d'éléments pertinents de la psychogenèse et de la sociogenèse des textes » (p. 86). La saisie de la matérialité langagière des textes littéraires permet ainsi de retracer le processus d'écriture à travers des éléments de repérage d'ordre discursif qui, dans une certaine mesure, reflètent les intentions du sujet écrivant ainsi que les conditions dans lesquelles s'exerce l'activité de production littéraire. La finalité de cette approche n'est ni la description de la langue ni l'essentialisation de celle-ci à travers le texte littéraire. Au contraire, son objectif est la mise en perspective de l'œuvre littéraire à travers la langue dans laquelle elle a été élaborée.

11 Même si beaucoup de travaux algériens s'en inspirent, peu d'entre eux se revendiquent explicitement de son héritage. Encore une fois, il est légitime de supposer que le souci de rester fidèle à la discipline de référence fait que toute tentative d'interdisciplinarité peut d'emblée être assimilée à une entreprise vouée à l'ambiguïté théorique. Les quelques travaux que nous avons consultés proposent des analyses qui n'abordent la question linguistique que dans un métadiscours métaphorique, construit en termes de rupture existentielle, de déchirement linguistique, de quête identitaire et d'engagement intellectuel. Sans être révélateurs de la tendance générale, ces travaux prennent le plus souvent la forme d'études de cas, consacrées à des œuvres ou à des auteurs en particulier. Dans l'une de ses publications, Amira Souames (2015) interroge l'identité du sujet écrivant dans l'œuvre d'Assia Djebar, où la langue française est utilisée pour dire un monde profondément façonné par l'oralité de l'arabe et du berbère. Le français ainsi déployé porte donc, en filigrane, les traces de ces deux langues, sans pour autant renoncer à sa syntaxe ni à sa grammaire. Même s'il n'intervient que dans cette langue, le sujet écrivant est bel et bien conscient de sa dimension plurilingue constitutive de son identité. Toujours en relation avec l'œuvre d'Assia Djebar, Houria Bensalem (2013) met en perspective la dimension linguistique par rapport à l'acte d'écriture, lui-même envisagé comme étant un investissement dans la mémoire et l'histoire, notamment celles des femmes de son pays de naissance qui n'ont accès qu'au code oral pour s'exprimer. Beaucoup d'écrivains algériens ont également fait l'objet de ce type d'approches. Citons le cas de Malek Haddad qui illustre bien l'idée selon laquelle la relation entre l'auteur et la langue d'écriture n'est pas 
toujours évidente. Ce dernier a connu, selon les études qui lui ont été consacrées, tout autant la fortune que l'infortune dans sa langue d'écriture, le français. D'après Goucem Nadira Khodja (2013), l'héritage colonial lié à la langue française a conduit cet auteur à se méfier de la culture que celle-ci véhicule, laquelle est considérée comme une culture de domination et d'aliénation, et c'est pourquoi elle est souvent contrecarrée par le recours à la langue des origines dans le processus d'écriture, donnant à l'occasion naissance à une langue dite « double » (Larbi, 2013).

Des exemples de ce type d'analyses sont multiples. Leur point commun est qu'ils se donnent davantage la peine d'étudier le prétexte linguistique que le contexte linguistique. Le prétexte se justifie par des considérations liées à l'intériorisation et à l'extériorisation de l'expérience langagière par le biais de l'écrit. Par contre, le contexte se démontre par l'analyse des faits qui gravitent autour de la praxis linguistique telle qu'elle est mise en œuvre dans et par le texte.

\section{Vers un tournant sociolinguistique}

12 Ce que nous appelons ici « tournant sociolinguistique » désigne la tendance qui consiste à rendre compte de la covariance entre langue et société à travers toutes les manifestations aussi bien formelles que symboliques de la création littéraire. Pendant longtemps, la langue a été considérée, dans son interaction avec la littérature, soit comme un système codifié ayant ses propres exigences lexicales et syntaxiques, soit comme un outil d'expression mis au service de la création. Cette conception binaire nous renvoie à deux écoles fondamentales, l'école structuraliste et l'école fonctionnaliste, qui, toutes deux, au-delà de leurs affinités épistémologiques manifestes, proposent des pistes de réflexion oscillant entre l'étude du bon usage et l'étude de l'usage tout court. C'est pourquoi, dans de nombreuses situations, la relation entre langue et littérature est posée uniquement en termes de construction, d'agencement scriptural, de grammaire textuelle et de style. La part socio-langagière inhérente à la production littéraire n'a été abordée que tardivement, notamment grâce à la mise en place d'un nouvel arsenal conceptuel issu de la sociolinguistique. A partir de là, un changement de paradigme est opéré, donnant lieu à des pratiques de recherche de plus en plus émancipées. Un article programmatique, co-signé par Aicha Kassoul et Mohamed-Lakhdar Maougal, est paru dans la revue Insaniyat, comme pour insister, une bonne fois pour toutes, sur le fait que «c'est au développement de la sociolinguistique que la critique littéraire [...] se doit d'avoir été déchargée d'un bavardage pseudo scientifique [...] auquel rares auront échappé les chercheurs les plus ou moins avertis » (2002: 74). Les questions inhérentes à l'usage littéraire de la langue ont dès lors le mérite d'être abordées loin de tout jugement esthétique et loin de tout préjugé idéologique.

13 Afin de mieux cerner les orientations de cette sociolinguistique de la littérature, rien ne nous semble plus approprié, du point de vue méthodologique, que de travailler sur un corpus qui donne accès aux discours produits par les chercheurs concernés par cette dynamique. Au cours de notre recherche documentaire, nous avons constaté deux événements éditoriaux en faveur de la recherche sociolinguistique orientée vers la littérature. Dans l'une de ses livraisons, la revue Cahiers de linguistique. Revue de sociolinguistique et de sociologie de la langue française (Blanchet et Taleb-Ibrahimi, 2009) a consacré toute une section, composée de trois articles, à la dimension sociolinguistique 
de la littérature algérienne. Un peu plus de trois ans plus tard, c'est au tour de la revue Socles de consacrer un numéro entier à l'interaction de la littérature avec les milieux plurilingues (Oucherif et Coste, 2013). Sur la base d'une sélection d'articles publiés dans ces revues, auxquels nous avons rajouté d'autres articles parus en varia dans d'autres revues, nous avons constitué un corpus destiné à être soumis à une analyse de contenu. Ce corpus se compose de neuf articles qui ont en commun le même ancrage théorique de référence, celui de la sociolinguistique.

L'analyse de contenu que nous proposons pour traiter notre corpus vise à dégager les axes de réflexion sur lesquels s'appuient les chercheurs dans leur démarche explicative ou compréhensive des faits de langue associés à la pratique littéraire. L'avantage de ce type d'analyse réside dans le fait qu'il guide l'exploration, l'organisation, la classification et la catégorisation des thèmes qui traversent le discours scientifique que nous avons sous les yeux. Patrick Chardenet explique que "[1]'analyse de contenu focalise sur la notion de thème qui constitue une affirmation sur un sujet [...] sous lequel un vaste réseau de formulations singulières peuvent être affectées " (2011:82). Cette technique consiste en effet à rendre compte de ce que disent les productions discursives organisées en corpus, en mettant en exergue la trame significative à l'œuvre. Une grille d'analyse a été conçue pour aiguiller notre travail, celle-ci ayant pour objectif de faire ressortir à partir de notre corpus les éléments suivants :

- l'approche adoptée en matière d'analyse ;

- les notions et les concepts théoriques mobilisés ;

- les références théoriques sollicitées ;

- la nature du corpus analysé ;

- et, le cas échéant, la conception du terrain.

Le tableau ci-dessous reprend les informations les plus pertinentes issues de l'exploration du contenu thématique de notre corpus :

Tableau 1 : Grille d'analyse appliquée au contenu des articles traitant de la relation entre langue et littérature dans une perspective sociolinguistique

\begin{tabular}{|c|c|c|c|c|c|}
\hline $\begin{array}{l}\text { Eléments } \\
\text { d'identification }\end{array}$ & $\begin{array}{l}\text { Approche } \\
\text { analytique }\end{array}$ & Notions et concepts & $\begin{array}{l}\text { Références } \\
\text { théoriques }\end{array}$ & Corpus & Terrain \\
\hline $\begin{array}{l}\text { Abbes-Kara } \\
(2006)\end{array}$ & $\begin{array}{l}\text { Approche } \\
\text { praxématique }\end{array}$ & $\begin{array}{l}\text { Diglossie, } \\
\text { pluriglossie, } \\
\text { discours sur la } \\
\text { langue, } \\
\text { minoration, } \\
\text { représentations }\end{array}$ & R. Lafont & $\begin{array}{l}\text { CEuvre } \\
\text { romanesque de } \\
\text { Mouloud } \\
\text { Mammeri }\end{array}$ & $\begin{array}{l}\text { Non } \\
\text { spécifié }\end{array}$ \\
\hline $\begin{array}{l}\text { Abbes-Kara } \\
(2009)\end{array}$ & $\begin{array}{l}\text { Analyse lexico- } \\
\text { statistique }\end{array}$ & $\begin{array}{l}\text { Hybridation, } \\
\text { néologisme, bi- } \\
\text { langue }\end{array}$ & $\begin{array}{l}\text { Non } \\
\text { spécifiées }\end{array}$ & $\begin{array}{l}\text { CEuvre } \\
\text { romanesque de } \\
\text { Mohamed Dib }\end{array}$ & $\begin{array}{l}\text { Non } \\
\text { spécifié }\end{array}$ \\
\hline $\begin{array}{l}\text { Abbes-Kara et } \\
\text { Kebbas (2013) }\end{array}$ & $\begin{array}{l}\text { Analyse } \\
\text { linguistique et } \\
\text { discursive }\end{array}$ & $\begin{array}{l}\text { Ecriture bilingue, } \\
\text { diglossie, discours } \\
\text { épilinguistique, } \\
\text { xénisme, emprunt, } \\
\text { calque, métissage }\end{array}$ & C. Lagarde & $\begin{array}{lr}\text { Cuvres } & \text { de } \\
\text { Mouloud } & \\
\text { Mammeri, Assia } \\
\text { Djebar } & \text { et } \\
\text { Mohamed Dib } & \end{array}$ & $\begin{array}{l}\text { Non } \\
\text { spécifié }\end{array}$ \\
\hline
\end{tabular}




\begin{tabular}{|c|c|c|c|c|c|}
\hline $\begin{array}{l}\text { Benbachir } \\
(2012)\end{array}$ & $\begin{array}{l}\text { Analyse } \\
\text { séquentielle de } \\
\text { la biographie } \\
\text { langagière }\end{array}$ & $\begin{array}{l}\text { Bilinguisme, } \\
\text { répertoire verbal, } \\
\text { diglossie }\end{array}$ & \begin{tabular}{|l|} 
Ch. \\
Perrégaux, \\
Ch. Deprez, J. \\
Gumperz
\end{tabular} & $\begin{array}{l}\text { Récit } \\
\text { autobiographique } \\
\text { der Soumya } \\
\text { Ammar-Khodja }\end{array}$ & $\begin{array}{l}\text { Non } \\
\text { spécifié }\end{array}$ \\
\hline $\begin{array}{l}\text { Benbachir } \\
(2017)\end{array}$ & $\begin{array}{l}\text { Analyse } \\
\text { thématique de } \\
\text { la biographie } \\
\text { langagière }\end{array}$ & $\begin{array}{l}\text { Plurilinguisme, } \\
\text { mobilité } \\
\text { linguistique }\end{array}$ & $\begin{array}{l}\text { A. Gohard- } \\
\text { Radenkovic, } \\
\text { E. Murphy- } \\
\text { Lejeune }\end{array}$ & $\begin{array}{l}\text { Entretiens } \\
\text { biographiques }\end{array}$ & $\begin{array}{l}\text { Non } \\
\text { spécifié }\end{array}$ \\
\hline $\begin{array}{l}\text { Chiraz et } \\
\text { Temim (2018) }\end{array}$ & Argotologie & $\begin{array}{l}\text { Argot, verlan, } \\
\text { pratiques } \\
\text { langagières, } \\
\text { fracture } \\
\text { linguistique }\end{array}$ & $\begin{array}{l}\text { J.-P. } \\
\text { Goudailler }\end{array}$ & $\begin{array}{l}\text { Le roman } \\
\text { Boumkœur de } \\
\text { Rachid Djaidani }\end{array}$ & $\begin{array}{l}\text { Allusion } \\
\text { au } \\
\text { terrain } \\
\text { urbain }\end{array}$ \\
\hline $\begin{array}{l}\text { Hamadache } \\
(2019)\end{array}$ & $\begin{array}{l}\text { Grille d'analyse } \\
\text { notionnelle }\end{array}$ & $\begin{array}{l}\text { Contact de langues, } \\
\text { bilinguisme, } \\
\text { diglossie, } \\
\text { alternance codique, } \\
\text { mélange de codes, } \\
\text { interférence } \\
\text { linguistique }\end{array}$ & $\begin{array}{lr}\text { M. L. } \\
\text { Clément, Ch. } \\
\text { Kossogorova }\end{array}$ & $\begin{array}{l}\text { Contes populaires } \\
\text { écrits en français }\end{array}$ & $\begin{array}{l}\text { Non } \\
\text { spécifié }\end{array}$ \\
\hline Kebbas (2009) & $\begin{array}{l}\text { Grille d'analyse } \\
\text { thématique }\end{array}$ & $\begin{array}{l}\text { Ecriture bilingue, } \\
\text { diglossie }\end{array}$ & C. Lagarde & $\begin{array}{l}\text { Æuvre } \\
\text { romanesque de } \\
\text { Mouloud } \\
\text { Mammeri }\end{array}$ & $\begin{array}{l}\text { Non } \\
\text { spécifié }\end{array}$ \\
\hline $\begin{array}{l}\text { Taleb- } \\
\text { Ibrahimi } \\
(2009)\end{array}$ & $\begin{array}{l}\text { Analyse } \\
\text { thématique }\end{array}$ & $\begin{array}{l}\text { Métissage, } \\
\text { bilinguisme }\end{array}$ & $\begin{array}{l}\text { Non } \\
\text { spécifiées }\end{array}$ & $\begin{array}{l}\text { Entretiens } \\
\text { journalistiques }\end{array}$ & $\begin{array}{l}\text { Non } \\
\text { spécifié }\end{array}$ \\
\hline
\end{tabular}

Les informations exposées dans ce tableau nous serviront à étayer notre analyse, notamment en ce qui concerne les modalités selon lesquelles se présente la sociolinguistique de la littérature dans le contexte universitaire algérien. Seront ainsi explicités les enjeux théoriques et les positionnements épistémologiques relatifs à son objet d'étude, à son corpus de prédilection et à son terrain d'investigation.

\section{Une conception sociolinguistique de la littérature}

Il y a lieu de noter que, dans leur majorité, les articles consultés jusque-là ne se risquent pas à donner une définition toute faite de la littérature, celle-ci n'étant bien évidemment pas leur objet d'étude de base. Le vague plane encore sur ce qu'est la littérature en sociolinguistique. Et pourtant, sur l'ensemble des textes étudiés, le mot « littérature » revient 48 fois $^{6}$. Quand il ne se présente pas seul, ce mot est associé soit à un qualificatif d'ordre géographique (littérature algérienne, littérature maghrébine), soit à un qualificatif d'ordre linguistique (littérature francophone, arabophone, etc.). Ce 
qui témoigne, de manière assez évidente, de la territorialisation de la littérature par le biais de la langue et, en même temps, de l'appropriation de la langue par la littérature.

Selon les propos de Khaoula Taleb-Ibrahimi, la littérature, du moins dans le contexte algérien, va de pair avec «la publication d'un nombre non négligeable de recueils poétiques, de nouvelles et de romans » (2009 : 239). Il s'agit donc d'un acte de création qui prend des formes multiples, où la prose côtoie les vers, et dont l'existence propre dépend de l'implication des instances éditoriales. Cette conception nous éloigne de l'univers du texte pour nous rapprocher un peu plus de celui de l'œuvre. Si le texte résulte de la somme des signes qui le composent et des procédés par lesquels il est construit, l'œuvre, par contre, nous renvoie à l'environnement dans lequel s'exerce l'acte d'écriture.

17 Attika-Yasmine Abbes-Kara en propose une autre définition formulée ainsi : « $[\mathrm{L}] \mathrm{a}$ littérature est une pratique langagière qui s'investit d'un contenu social, qu'elle est également dialogique du fait qu'il n'y a pas de message tout à fait transmis par l'un et l'autre mais une interaction verbale " (2006:27). A partir de là, l'accent est mis sur le fait que la littérature fait interagir des formes linguistiques avec un contenu social. En tant que pratique, elle implique une instance auctoriale, une assise textuelle, un ancrage contextuel et, au bout de la chaine, une instance réceptrice. Tous ces éléments sont appelés à entrer dans une relation dialogique les uns avec les autres. Le texte devient ainsi indissociable du contexte dans lequel il a été conçu ainsi que du contexte qu'il conçoit. Il en va de même pour l'auteur et le lecteur qui se laissent, l'un comme l'autre, entrainer dans une forme de communication interactive qui n'est pas forcément traçable.

18 Alors, contrairement à la glottocritique qui s'intéresse à la littérature à travers la langue, la sociolinguistique emprunte le chemin inverse, en ce sens qu'elle s'intéresse à la langue à travers la littérature. Et là encore, il faut préciser qu'elle ne s'intéresse pas à la langue dans sa forme et encore moins à travers ses mécanismes de déploiement au fil de la plume. Elle s'y intéresse à travers la dimension sociale et factuelle que son usage en littérature laisse entrevoir.

\section{Caractéristiques sociolinguistiques des corpus littéraires}

19 Indépendamment de sa nature générique, tout texte littéraire recèle, aussi bien en dedans qu'en dehors, des phénomènes langagiers susceptibles d'être examinés à la lumière des apports théoriques de la sociolinguistique. Dans une certaine mesure, les corpus littéraires véhiculent un potentiel documentaire qui renseigne sur des pratiques linguistiques visibles et lisibles dans et/ou à travers le texte. Dans le texte sont repérables les procédés liés à l'usage de la langue à des fins littéraires. A travers le texte sont repérables les normes et les attitudes qui sous-tendent l'usage en question.

Il est à remarquer que, dans les travaux que nous avons consultés, le débat ne tourne plus autour de ce qu'est la langue en littérature ni de ce qu'est la langue pour la littérature. Il se trouve que ces questions, comme nous l'avons déjà mentionné plus haut, ont largement été abordées dans des recherches antérieures d'inspiration glottocritique. C'est désormais la nature discursive des corpus littéraires qui interpelle les chercheurs versés en sociolinguistique, et c'est aussi le contexte des œuvres 
littéraires qui leur donne à réfléchir, notamment sur la relation entretenue, en amont et en aval, par le sujet écrivant avec sa langue d'écriture ainsi qu'avec les éventuelles langues de son répertoire verbal (Benbachir, 2012; 2017). La production littéraire a comme point de départ la position à partir de laquelle l'instance auctoriale signe ses écrits et comme point d'arrivée la position occupée par l'instance réceptrice. Cela explique la tendance de certains chercheurs à intégrer dans leurs travaux les discours tenus en dehors du texte, mais qui sont dialogiquement reliés au texte. Ainsi, les déclarations publiques et les interventions médiatiques des auteurs sont, comme le démontre Khaoula Taleb-Ibrahimi (2009), porteuses de renseignements d'ordre sociolinguistique qui expliquent les choix littéraires effectués par les uns et les autres.

21 Afin de vérifier l'hypothèse selon laquelle les multiples écrits d'un seul auteur ou les écrits d'un collectif d'auteurs appartenant à la même génération se télescopent et se recoupent par endroits, certains chercheurs, à l'instar de Attika-Yasmine Abbes-Kara et Malika Kebbas (2013) privilégient d'interroger plusieurs œuvres à la fois dans l'espoir de dégager des pratiques linguistiques en rapport avec un mode de socialisation langagière influencé par le contexte tant social que littéraire. Qu'il s'agisse du contexte colonial ou du contexte postcolonial, les usages linguistiques dans la littérature algérienne portent les traces d'une pluralité linguistique différemment mise en texte.

La mise en texte de la pluralité linguistique donne à voir des phénomènes qui s'inscrivent dans le registre du contact des langues (Hamadache, 2019), de la mobilité linguistique (Benbachir, 2017) ou de la créativité langagière (Chiraz et Temim, 2018). Les concepts de bilinguisme, de diglossie et d'alternance codique ont ceci d'original qu'ils mettent l'accent sur les rapports instaurés entre les langues dans et par la littérature. Dans le même ordre d'idées, l'analyse des discours épilinguistiques (AbbesKara, 2006; Kebbas, 2009), c'est-à-dire ceux tenus sur les langues au sein même des écrits littéraires, permet de mettre en évidence la spécificité des rapports entretenus à l'égard des langues par le biais de la composition littéraire.

\section{La littérature comme terrain d'investigation}

La sociolinguistique a pour vocation de se saisir de la langue en acte, et c'est la raison pour laquelle elle a longtemps été conçue comme une "linguistique de terrain " (Blanchet, 2012). Une chose est sûre est que la littérature n'est pas directement l'objet d'étude de la sociolinguistique; elle n'en est qu'un terrain d'investigation. Cette affirmation nous invite à reconsidérer la notion de terrain et à en expliquer les particularités heuristiques.

Des neuf articles que nous avons analysés, aucun d'eux ne fait explicitement référence à la notion de terrain. Par contre, la notion de contexte y est fréquemment évoquée. Ce n'est qu'au prix d'un effort de déduction qu'il est possible d'apercevoir entre les lignes le reflet d'un terrain à peine circonscriptible. Se voulant assez illustratif à ce sujet, l'article de Hakim Chiraz et Dalida Temim (2018) met en relation, à travers l'analyse du roman Boumkour de Rachid Djaidani, les pratiques langagières dites argotiques avec le terrain urbain au sein duquel elles surgissent.

A vrai dire, ce n'est pas le terrain en lui-même qui est complètement aboli dans ces études, mais c'est plutôt l'enquête de terrain, au sens ethnographique du terme, qui est délaissée au profit d'une investigation de type documentaire. Aux corpus traditionnellement sollicités se sont succédé des corpus prêts à l'emploi, ceux-ci étant 
directement puisés dans des œuvres littéraires de divers genres ou dans des entretiens accordés par des auteurs à la presse.

Dans l'absolu, il n'est pas exclu que la «sociolinguistique [soit] appliquée à un objet pour lequel un terrain n'est guère envisageable, ou bien change complètement de sens " (Gasquet-Cyrus, 2015: 20). Nous sommes exactement dans ce cas de figure avec la sociolinguistique de la littérature, dans la mesure où le terrain ne correspond plus ici à un espace géographique susceptible d'être parcouru à l'aide d'une méthodologie artisanale. Dans cette perspective, la littérature représente à elle seule un espace de création multiforme impliquant une série d'interactions entre divers acteurs situés à des niveaux différents sur la chaine de production, de diffusion et de consommation. En tant que telle, la littérature est aussi le siège de libertés et de contraintes, observables dans le texte et en dehors du texte. Et c'est à ce titre, justement, qu'elle se prête au jeu de l'exploration et de l'investigation sociolinguistique.

$\mathrm{Au}$ terme de cette réflexion, il convient de souligner qu'en réponse aux questions que nous nous sommes posées au tout début, nous avons de fil en aiguille abouti à ces conclusions :

- la littérature est envisagée en sociolinguistique comme une pratique étroitement liée à un contexte de production et de circulation, et surtout comme une pratique qui fait interagir des formes linguistiques fluctuantes avec un contenu socialement marqué ;

- la littérature est le siège de phénomènes sociolinguistiques qui se déploient sur deux principaux axes, celui des rapports instaurés entre les langues et celui des rapports entretenus à l'égard des langues ;

- la littérature n'est pas à proprement parler un objet d'étude pour la sociolinguistique mais un terrain d'investigation à entrée textuelle.

Ces trois conclusions sont révélatrices des présupposés épistémologiques qui guident les travaux effectués dans le cadre de la sociolinguistique de la littérature. L'intérêt d'une telle approche réside dans le fait que celle-ci contribue, par-delà les frontières disciplinaires traditionnelles, à une meilleure compréhension des relations complexes entre langue et littérature.

\section{BIBLIOGRAPHIE}

ABBES-KARA, Attika-Yasmine, « Le roman algérien en situation de pluriglossie », buhut simya'iya, 1/2, 2006, pp. 27-37. Disponible sur [https://www.asjp.cerist.dz/en/article/26108] (consulté le $24 / 12 / 2020)$

ABBES-KARA, Attika-Yasmine, « Du métissage littéraire au métissage culturel et linguistique. Le cas de Mohamed Dib », Cahiers de linguistique. Revue de sociolinguistique et de sociologie de la langue française, 34/1, 2009, pp. 217-226.

ABBES-KARA, Attika-Yasmine, KEBBAS, Malika, « Pour une problématisation des "écritures bilingues" dans la littérature francophone algérienne », Socles, 2, 2013, pp. 21-36. Disponible sur [https://www.asjp.cerist.dz/en/article/2636] (consulté le 19/07/2020) 
BENBACHIR, Naziha, «Le bilinguisme dans le récit autobiographique de Soumya AmmarKhodja », Revue Annales du patrimoine, 12, 2012, pp. 7-17. Disponible sur [https:// www.asjp.cerist.dz/en/article/39193] (consulté le 03/01/2021)

BENBACHIR, Naziha, «L'expérience plurilingue chez les écrivains du bassin méditerranéen : une cartographie des langues et des mobilités », Insaniyat, 77-78, 2017, pp. 123-140. Disponible sur [https://www.asjp.cerist.dz/en/article/87572] (consulté le 25/07/2020)

BENSALEM, Houria, «La problématique de la langue et de l'écriture chez Assia Djebar », El-khitab, 16, 2013, pp. 271-276. Disponible sur [https://www.asjp.cerist.dz/en/article/21682] (consulté le 13/03/2020)

BERNABE, Jean, « Contribution à une approche glottocritique de l'espace littéraire antillais », La Linguistique, 18/1, 1982, pp. 85-109.

BLANCHET, Philippe, La linguistique de terrain, méthode et théorie. Une approche ethnosociolinguistique de la complexité, PUR, Rennes, 2012.

BLANCHET Philippe, «Pensée complexe ou objet complexe ? Sur les enjeux épistémologiques de la complexité en linguistique et sociolinguistique », Cahiers internationaux de sociolinguistique $\mathrm{n}^{\circ} 7$, 2015, pp. 57-74.

BLANCHET, Philippe \& TALEB-IBRAHIMI, Khaoula (dir.), « Le plurilinguisme maghrébin : comparaison de pratiques sociales ordinaires, techniques, didactiques et littéraires en Algérie, au Maroc et en Tunisie ", Cahiers de linguistique. Revue de sociolinguistique et de sociologie de la langue française, 34/1, 2009.

BOUTET, Josiane, La vie verbale au travail. Des manufactures aux centres d'appels, Octares, Toulouse, 2008.

CALVET, Louis-Jean, Les voix de la ville, introduction à la sociolinguistique urbaine, Payot, Paris, 1994.

CHARDENET, Patrick, « Les principales méthodes et leurs techniques de construction des observables. L'échange avec les acteur comme méthode de production des données [entretiens et groupes de discussion] ", dans BLANCHET, Philippe, CHARDENET, Patrick (dir.), Guide pour la recherche en didactique des langues et des cultures. Approches contextualisées, AUF/Editions des archives contemporaines, Paris, 2011, pp. 77-91.

CHIRAZ, Hakim \& TEMIM, Dalida, « L'argot des jeunes des cités dans le roman Boumkœur de Rachid Djaidani », Revue algérienne des sciences du langage, 3/1, 2018, pp. 101-113. Disponible sur [https://www.asjp.cerist.dz/en/article/59478] (consulté le 05/01/2021)

DABENE, Louise, Repères sociolinguistiques pour l'enseignement des langues. Les situations plurilingues, Hachette, Paris, 1994.

GADET, Françoise, La variation sociale en français, Editions Ophrys, Paris, 2007.

GASQUET-CYRUS, Médéric, «"Je vais et je viens entre terrains" Réflexions sur le terrain dans la théorisation sociolinguistique ", Langage et Société, 154, 2015, pp. 17-32.

GAUVIN, Lise, La fabrique de la langue. De François Rabelais à Réjean Ducharme, Editions du Seuil, Paris, 2004.

GUMPERZ, John, Engager la conversation. Introduction à la sociolinguistique interactionnelle, Minuit, Paris, 1989.

HAMADACHE, Tahar, « Contacts de langues et/ou de cultures dans les contes populaires écrits en langue française », Multilinguales, 7/2, 2019, pp. 166-189. Disponible sur [https:// www.asjp.cerist.dz/en/article/109956] (consulté le 26/12/2020) 
KASSOUL, Aicha \& MAOUGAL, Mohamed-Lakhdar, « Actancialiser ou déconstruire », Insaniyat, 17-18, 2002, pp. 71-77.

KEBBAS, Malika, « Sociolinguistique et littérature algérienne francophone. L'écriture bilingue dans l'œuvre romanesque de Mouloud Mammeri ", Cahiers de linguistique. Revue de sociolinguistique et de sociologie de la langue française, 34/1, 2009, pp. 227-238.

KHODJA, Goucem Nadira, « Une écriture entre deux langues. Contraintes et enjeux de l'entredeux chez Malek Haddad », Socles, 2, 2013, pp. 97-108. Disponible sur [https://www.asjp.cerist.dz/ en/article/2641] (consulté le 19/07/2020)

LARBI, Chérifa, «La langue "double" chez Malek Haddad », Socles, 2, 2013, pp. 109-119. Disponible sur [https://www.asjp.cerist.dz/en/article/2642] (consulté le 19/07/2020)

MORIN, Edgar, Introduction à la Pensée Complexe, Paris, ESF, 1990.

OUCHERIF, Lamia \& COSTE, Claude (dir.), « L'invention d'une langue littéraire dans un milieu plurilingue », Socles, 2, 2013.

PIAGET, Jean, Logique et connaissance scientifique, Gallimard, Paris, 1967.

SIMONIN, Jacky \& WHARTON, Sylvie, « Sociolinguistique des contacts de langues. Un domaine en plein essor », dans SIMONIN Jacky \& WHARTON Sylvie (dir.), Sociolinguistique du contact.

Dictionnaire des termes et concepts, ENS Editions, Lyon, 2013, pp. 13-18.

SOUAMES, Amira, «Identité du sujet écrivant au carrefour des langues dans l'œuvre d'Assia Djebar ", Al-bâhith, 14, 2015, pp. 12-25. Disponible sur [https://www.asjp.cerist.dz/en/article/ 25266] (consulté le 19/07/2020)

TALEB-IBRAHIMI, Khaoula, « Ecrire dans la langue de l'autre, écrire entre les langues. Plaidoyer pour le métissage littéraire ", Cahiers de linguistique. Revue de sociolinguistique et de sociologie de la langue française, 34/1, 2009, pp. 239-246.

\section{NOTES}

1. Il faut entendre ici par épistémologie ce que Jean Piaget décrit comme étant «l'étude de la constitution des connaissances valables » $(1967: 6)$. Alors que le savoir en tant qu'ensemble de propositions à valeur synthétique ou analytique, organisées en notions et en concepts, nous renvoie à la théorie, le savoir tel qu'il est pensé et élaboré nous renvoie à l'épistémologie.

2. L'ASJP (Algerian Scientific Journal Platform) est un portail électronique destiné à la mise en ligne du contenu des revues scientifiques affiliées à des établissements universitaires algériens. Placé sous la responsabilité du Centre de recherche sur l'information scientifique et technique (CERIST), ce portail est en libre accès via le lien : [https://www.asjp.cerist.dz]. Les données qu'il permet d'explorer témoignent de la dynamique scientifique ayant cours, notamment en matière production, dans le contexte algérien.

3. La recherche par mots-clés a été effectuée en français. Cette manière de procéder ne présente aucun risque quant à la fiabilité des résultats obtenus, puisque, indépendamment de la langue de rédaction utilisée dans le corps du texte, la plupart des revues algériennes exigent que les résumés et les mots-clés associés aux articles publiés soient notés en français.

4. L'identification de ces champs de recherche repose ici sur leur dénomination institutionnelle.

5. Pour plus de détails sur ces chiffres, voir Annexes.

6. Le calcul des occurrences a été effectué par l'intermédiaire du logiciel Tropes (version 8.4.4). Précisons encore que l'adjectif «littéraire » est, pour sa part, mentionné dans 49 endroits différents dans le corpus, en référence à une activité de production à caractère scriptural. 


\section{RÉSUMÉS}

L'objectif de cet article est de reconstituer, à partir de quelques travaux scientifiques algériens, les fondements épistémologiques d'une sociolinguistique de la littérature qui se veut en pleine émergence. Dans un premier temps, nous avons tenu à souligner la prédisposition de la sociolinguistique à travailler sur des terrains hétérogènes, juste parce que la question de l'usage de la langue y est posée. Nous avons ensuite, à l'aide d'une étude quantitative basée sur des données bibliométriques, fait en sorte de situer les travaux sociolinguistiques orientés vers la littérature par rapport à l'ensemble de la production scientifique qui traite de la relation entre langue et littérature. Enfin, nous avons approfondi notre analyse par une étude qualitative portant sur le contenu des publications qui s'inscrivent dans le cadre de la sociolinguistique de la littérature.

On the basis of Algerian research studies, this article aims to reconstitute the epistemological underpinnings of the sociolinguistics of literature, whose emergency is in full swing. First, the researcher highlights the tendency of sociolinguistics to cover heterogeneous fields, given that it raises the question of language usage. Furthermore, a quantitative approach, based on bibliometric data, was adopted to examine literature-oriented sociolinguistics in relation to academic publications that study the nexus between language and literature. Finally, the researcher broadened the analysis through a qualitative study of the content of academic work which fall under which fall within the framework of the sociolinguisitcs literature.

\section{INDEX}

Mots-clés : épistémologie, glottocritique, langue, littérature, sociolinguistique Keywords : epistemology, glottocriticism, language, literature, sociolinguistics

\section{AUTEURS}

\section{MOKHTAR BOUGHANEM}

Université d'Alger 2, Laboratoire LISODIL, Algérie

\section{HASSIBA BENALDI}

Université d'Alger 2, Laboratoire LISODIL, Algérie 